ARTICLE

\title{
Structural basis for tuning activity and membrane specificity of bacterial cytolysins
}

\author{
Nita R. Shah", Tomas B. Voisin ${ }^{1}$, Edward S. Parsons ${ }^{2}$, Courtney M. Boyd ${ }^{1}$, Bart W. Hoogenboom (D) ${ }^{2,3}$ \&
} Doryen Bubeck (D) ${ }^{1 \times}$

Cholesterol-dependent cytolysins (CDCs) are pore-forming proteins that serve as major virulence factors for pathogenic bacteria. They target eukaryotic cells using different mechanisms, but all require the presence of cholesterol to pierce lipid bilayers. How CDCs use cholesterol to selectively lyse cells is essential for understanding virulence strategies of several pathogenic bacteria, and for repurposing CDCs to kill new cellular targets. Here we address that question by trapping an early state of pore formation for the CDC intermedilysin, bound to the human immune receptor CD59 in a nanodisc model membrane. Our cryo electron microscopy map reveals structural transitions required for oligomerization, which include the lateral movement of a key amphipathic helix. We demonstrate that the charge of this helix is crucial for tuning lytic activity of CDCs. Furthermore, we discover modifications that overcome the requirement of cholesterol for membrane rupture, which may facilitate engineering the target-cell specificity of pore-forming proteins.

\footnotetext{
${ }^{1}$ Department of Life Sciences, Sir Ernst Chain Building, Imperial College London, London SW7 2AZ, UK. ${ }^{2}$ London Centre for Nanotechnology, University College London, London WC1H OAH, UK. ${ }^{3}$ Department of Physics and Astronomy, University College London, Gower Street, London WC1E 6BT, UK.

凶email: d.bubeck@imperial.ac.uk
} 
P ore-forming proteins rupture lipid bilayers to kill target cells. They comprise the largest class of virulence factors for pathogenic bacteria and are prevalent in all kingdoms of life $^{1}$. Cholesterol-dependent cytolysins (CDCs) are pore-forming proteins secreted by more than five genera of Gram-positive bacteria, including human pathogens from Streptococcus, Clostridium, and Listeria. Pore-forming proteins also play a crucial role in immune defence, killing Gram-negative bacteria, cancer cells, and phagocytosed microbes ${ }^{2}$. Understanding how these proteins discriminate between self-cells and target membranes will provide insight into fundamental virulence strategies, as well as facilitate the application of engineered CDCs that lyse new cellular targets.

Many pore-forming proteins depend on lipid specificity to select their targets. For example, the immune protein perforin preferentially targets post-synaptic membranes through lipid disorder phases and neutral charge headgroups ${ }^{3}$. Another immune protein, gasdermin, specifically binds cardiolipin and phosphoinositide lipids to direct activity against mitochondria and the inner leaflets of eukaryotic plasma membranes, triggering cell death ${ }^{4,5}$. For CDCs, the requirement of cholesterol directs lytic activity towards plasma membranes of eukaryotic hosts ${ }^{6}$. Since cholesterol is not present in bacterial cells that secrete CDCs, this is one way the pathogen protects itself from damage during infection and toxin production; it also limits the repurposing of CDCs to attack bacterial pathogens. Many CDCs, such as pneumolysin (PLY), bind cholesterol directly through a conserved cholesterol-recognition motif located on a membranebinding loop ${ }^{7}$. Although cholesterol is a well-known receptor for PLY, it may not be the only one. PLY also binds to a mannose receptor in dendritic cells, downregulating inflammation and promoting bacterial survival ${ }^{8}$. Other CDCs, such as intermedilysin (ILY) secreted by Streptococcus intermedius, achieve species-specificity for their hosts by hijacking the cell surface receptor CD59 to initiate membrane binding 9 . For this subgroup of CDCs, the interaction with CD59 is sufficient for attachment and the role of cholesterol is restricted to pore formation ${ }^{10}$. While differences in membrane targeting may reflect diversity in CDC virulence strategies, lipid dependency for membrane penetration remains highly conserved.

CDCs bind target membranes through interactions located in their domain 4 (D4). Both the cholesterol-recognition loops of $\mathrm{PLY}^{7}$ and CD59-binding site of ILY lie within this domain ${ }^{9}$. A long extended domain 2 (D2) flexibly links D4 to the membrane attack complex perforin (MACPF)/CDC domain (comprised of D1 and D3 together), which is responsible for pore formation ${ }^{11}$. Membrane binding of CDC monomers triggers a series of conformational rearrangements that are required for oligomerization into an assembly referred to as a prepore. A dramatic vertical collapse of the oligomeric prepore brings two helical bundles (HB1 and HB2) within the MACPF/CDC domain close to the target membrane. $\mathrm{HB} 1$ and $\mathrm{HB} 2$ residues undergo rearrangement in secondary structure to form adjacent transmembrane $\beta$-strands in the pore ${ }^{12-15}$. These rearrangements are facilitated by stabilizing interactions between a number of amino acids at the MACPF/CDC domain interface ${ }^{16}$, but structural details of intermediate conformations remain unresolved.

Here we report a structure of an early prepore-trapped conformation of the CDC ILY solved by cryo electron microscopy (cryoEM). ILY binds the human immune receptor CD59 as a monomer to initiate attachment to the membrane. CD59 binding then promotes ILY oligomerization and triggers movement of a highly conserved amphipathic helix that encodes activity and lipid specificity of CDC pore formation. This helix can be modified to create cholesterol-independent CDCs or "super-CDCs" with significantly enhanced activity. Hence, our results provide a blueprint for engineering broad-purpose pore-forming proteins with tunable lytic activity.

\section{Results}

CryoEM reconstruction of an ILY early prepore. ILY from Streptococcus intermedius targets human cells by binding the GPIanchored complement regulator $\mathrm{CD}^{2} 9^{9}$ through an extended $\beta$ hairpin of $\mathrm{D} 4^{17}$. To understand how this interaction initiates oligomerization on a target membrane, we trapped ILY in an early prepore state using a disulfide lock that restricts movement between D2 and the MACPF/CDC domain ${ }^{10}$. This disulfidelocked ILY variant binds CD59 and forms SDS-sensitive loosely associated oligomers ${ }^{10}$, analogous to previously characterized CDC early prepore states ${ }^{18}$. We then used cryo-electron microscopy (cryoEM) to visualize this ILY variant in complex with CD59 anchored to cholesterol-containing lipid nanodiscs. We collected data on a Titan Krios microscope equipped with a direct electron detector and processed images using RELION ${ }^{19}$. 2D classification revealed heterogeneity in the number of subunits within a single nanodisc (Supplementary Fig. 1). Extensive 2D and $3 \mathrm{D}$ classification resulted in a final reconstruction comprised of 51,041 particles. The data were further refined with local symmetry, resulting in a map with an average resolution of $4.6 \AA$. (Supplementary Fig. 2). The final local resolution-sharpened density map illustrates an arc of five ILY-CD59 complexes, with the central subunit best resolved (Fig. 1a, Supplementary Fig. 1). We therefore built a model into the density of the central subunit, which served as the basis for refinement of a three-subunit oligomer (Fig. 1a and Supplementary Fig. 3).

Our cryoEM map reveals an oligomeric complex whereby a single CD59 can interact with two neighboring ILY monomers (ILY and ILY'). Similar to the soluble monomeric ILY-CD59 crystal structures $^{17,20}$, a $\beta$-hairpin of one ILY D4 extends the central $\beta$-sheet of CD59. The same $\beta$-hairpin of an adjacent monomer (ILY'), together with the tip of D2, sandwiches an $\alpha$ helix of CD59 to form the second interface (Fig. 1b). This interface is dominated by two CD59 residues decorated with Olinked glycans in the native protein ${ }^{21}$. ILY binds to an O-glycan prevalent on human $\mathrm{CD} 59^{22}$, suggesting that sugar recognition may play a role in oligomerization and could be engineered to impact target-cell specificity.

CD59 binding initiates the secondary structure rearrangement of the MACPF/CDC outer $\beta$-strand $(\beta-5)$. In our structure, residues within the $\beta-5$ strand form a new helix that comprises part of a helix-turn-helix motif (Fig. 1c, Supplementary Fig. 4, and Supplementary Fig. 5). As a result, the $\beta-4$ strand is exposed to propagate oligomerization by binding the $\beta-1$ strand of a neighboring monomer. In the early prepore, $\beta-4$ rotates from its position in the monomeric initiation complex to reveal an intermediate transition along the pore formation pathway (Supplementary Fig. 5). The ILY prepore assembly is most likely further stabilized by complementary charges on neighboring helical faces of the helix-turn-helix motif (Fig. 1c). Similarly, alternating charges of this motif in the PLY pore are thought to stabilize an inner barrel of helices above the membrane ${ }^{15}$.

In our complex, ILY extends to a vertical height of $110 \AA$, revealing an oligomeric prepore that has not yet collapsed towards the target membrane. Specifically, residues of the two MACPF/CDC helical bundles (HB1 and HB2) that eventually form transmembrane $\beta$-hairpins in the pore assembly do not contact the bilayer. Helical bundle 2 (HB2) is comprised of a vertical helix and a horizontal helix (h-helix) (Fig. 1d and Supplementary Fig. 4), which is directly N-terminal to the newly positioned MACPF $\beta-4$ strand (Supplementary Fig. 5). Compared with the monomeric ILY-CD59, we observe a lateral shift in these 
a
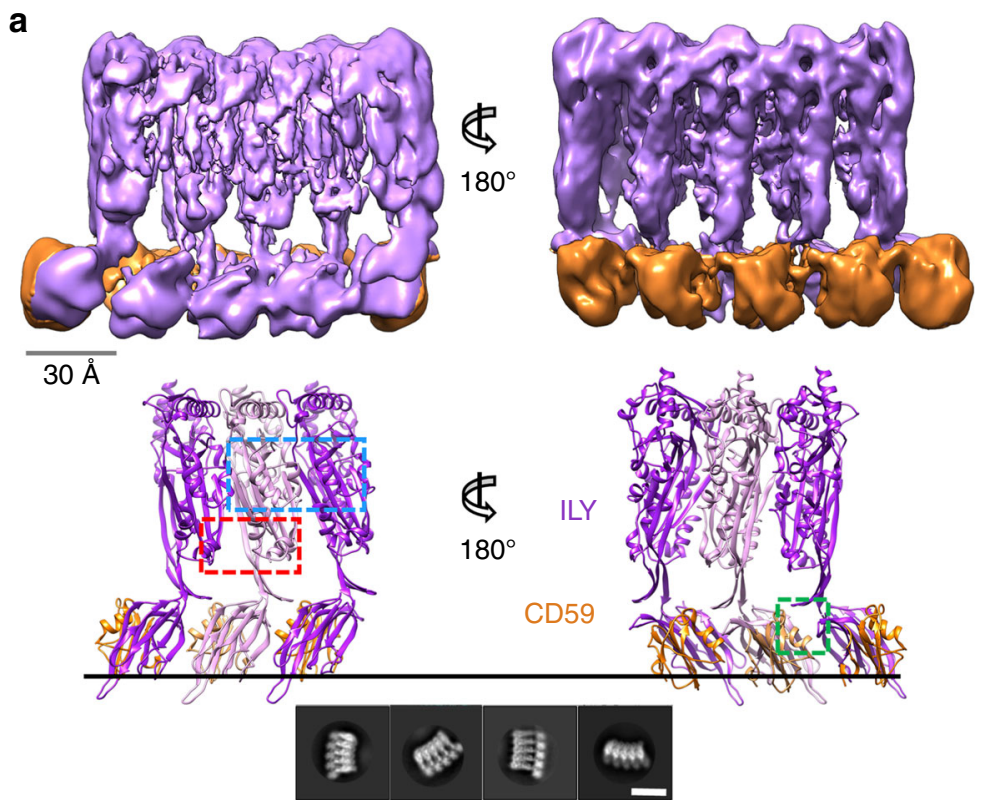

d

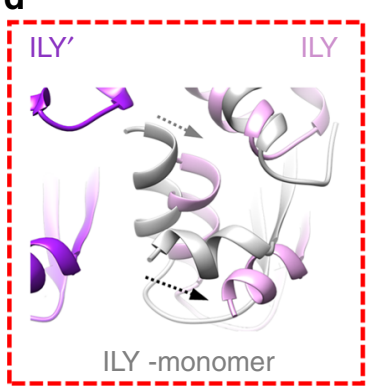

e

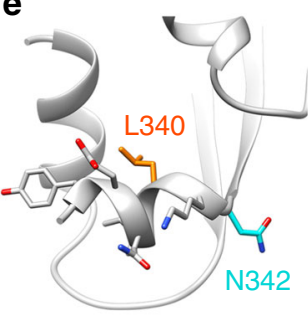

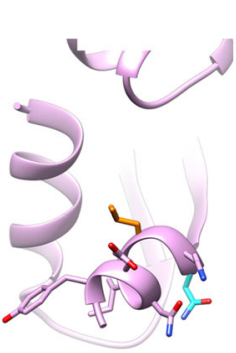

b
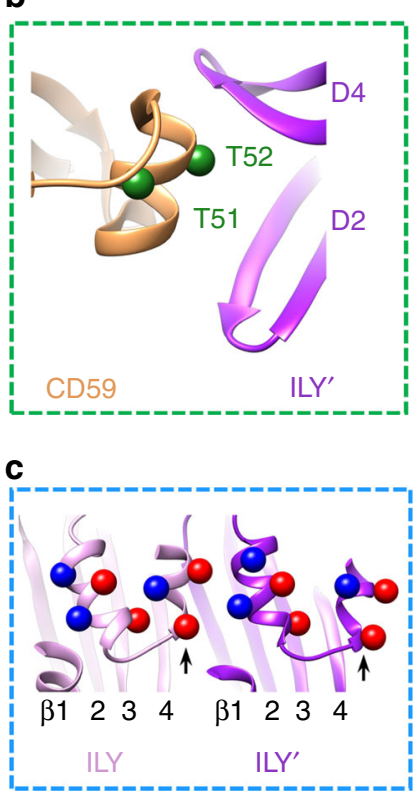

f

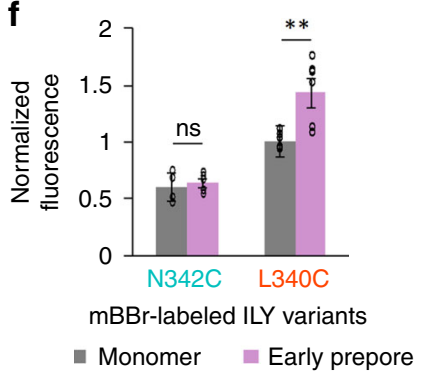

Fig. 1 Structure of an ILY-CD59 early prepore oligomer. a CryoEM reconstruction filtered according to local resolution (top) and structural model derived from the cryoEM density map (bottom). In the model, adjacent ILY monomers (ILY and ILY') are pink and purple, respectively. Neighboring CD59 molecules are orange. Black line represents the membrane surface. Dashed boxes indicate regions highlighted in panels (b) (green), (c) (blue), and (d) (red). Bottom inset shows a selection of 2D class averages. White scale bar, $100 \AA$. b Interaction interface between CD59 and domains 2 and 4 (D2, D4) of neighboring ILY'. Green spheres indicate positions of residues modified by O-linked glycans in endogenous CD59 (T51, T52). c Helix-turn-helix motif of the oligomeric early prepore. Newly formed helix of ILY (formerly $\beta$-strand 5) is indicated by black arrow. Spheres indicate negative (red) and positive (blue) charged residues within the helices. $\beta$-strands of the MACPF/CDC domain are labeled. $\mathbf{d}$ Superposition of the early prepore ILY (pink) with monomeric ILY (gray) from crystal structure PDBID: 4BIK ${ }^{17}$. Gray and black arrows indicate direction of shifts for the vertical and horizontal helix (h-helix), respectively. Neighboring ILY' is shown for reference. e Structural model showing the location of residues L340 (orange) and N342 (cyan) modified by the fluorescent tag, mBBr. Sidechains have been added in $\mathrm{COOT}^{44}$ for visualization purposes. $\mathbf{f}$ Normalized fluorescence intensity of mBBr-labeled h-helix residues in monomeric (gray) and oligomeric early prepore (pink) ILY. Individual data points from six independent experimental assays are shown as circles, error bars represent standard deviation with the center of the bar indicating the mean value, $p$-value significance determined by a two-sided Student's $t$ test: ns, not significant, $p=0.19 ;{ }^{\star \star} p=0.0060$.

helices upon oligomerization (Fig. 1d and Supplementary Fig. 4). This movement prevents an otherwise steric clash with a neighboring monomer and allows the oligomer to propagate. Given the moderate resolution of our map, we sought to verify the movement of these helices using a fluorescence-based assay. To investigate how oligomerization influences the local chemical environment of the h-helix, we covalently linked monobromobimane $(\mathrm{mBBr})$ to residues on either the bottom (membrane adjacent) or top face of the h-helix in early prepore-locked ILY (Fig. 1e). Fluorescence was then measured in solution (monomer) or in the presence of CD59-containing DOPC:cholesterol liposomes (early prepore). Upon oligomerization, we observed an increase in $\mathrm{mBBr}$ fluorescence when the fluorophore was tethered to the top face of the h-helix, consistent with movement of L340 as it packs more closely against the surrounding protein. By contrast, the fluorescence intensity remained the same for labels attached to the bottom face of the h-helix, in agreement with our structural data showing that the packing of membraneadjacent residues is not affected by this conformational change (Fig. 1e, f). Together, these biochemical data corroborate the structurally observed lateral movement of the HB2 helices.

The h-helix encodes the ability to tune lytic activity. Based on our structure, we hypothesized that the h-helices of a collapsed oligomer could play a role in destabilizing the lipid bilayer. To test this, we first needed to stall pore formation of a collapsed oligomer by trapping ILY in a late prepore assembly intermediate. We generated disulfide-locked ILY mutants to restrict movement of either HB1 (HB1-lock) or HB2 (HB2-lock) and prevent the formation of transmembrane $\beta$-hairpins (Fig. 2a and Supplementary Fig. 6). SDS gel shift assays reveal a portion of $\mathrm{HB1}$ and 
a

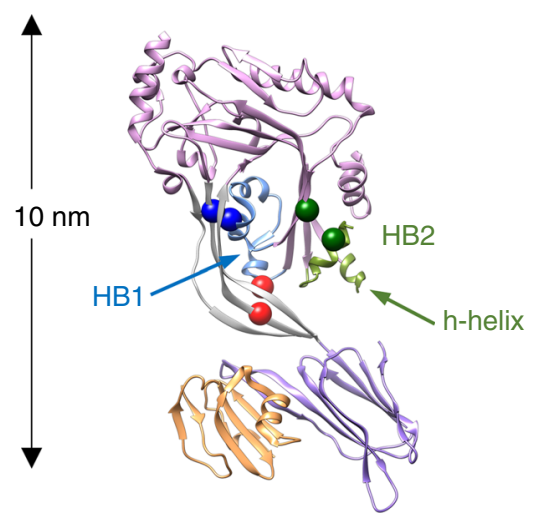

C
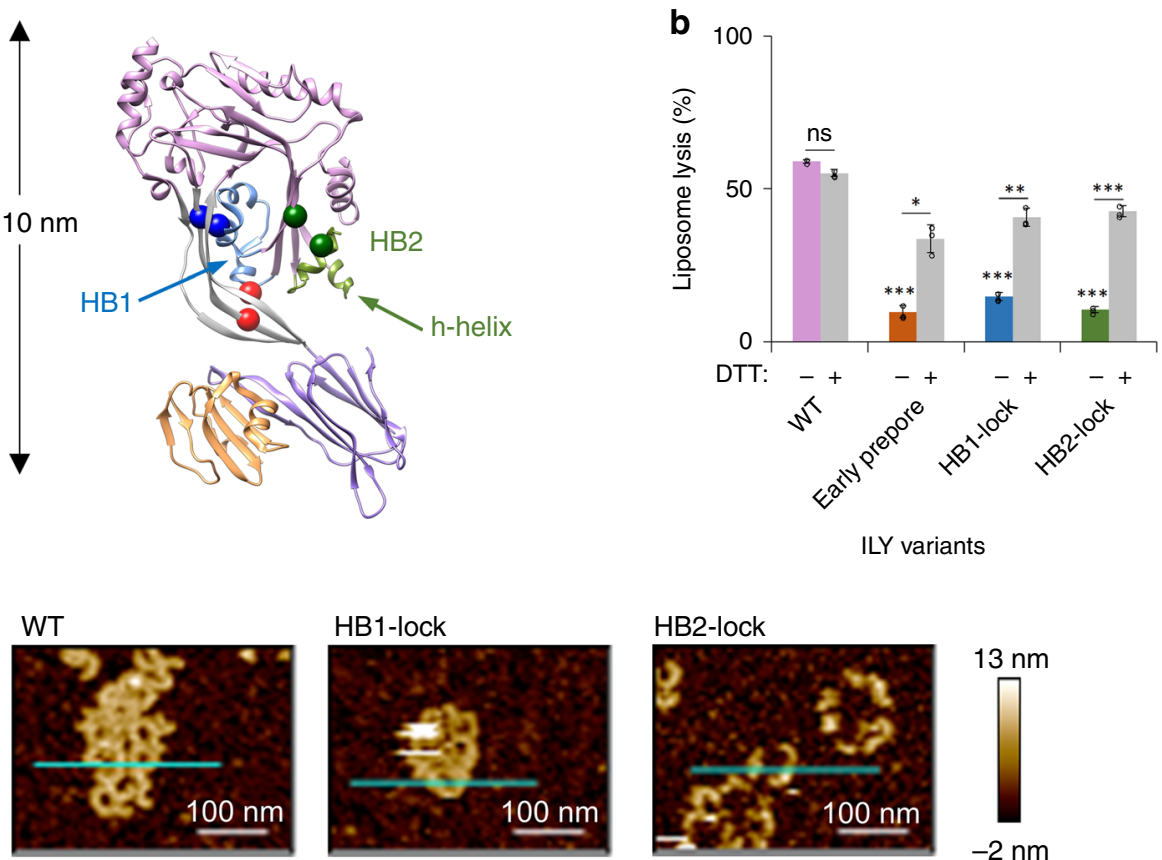

ILY variants

\section{HB2-lock}

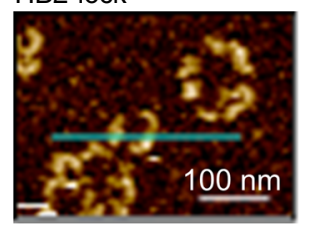

$13 \mathrm{~nm}$
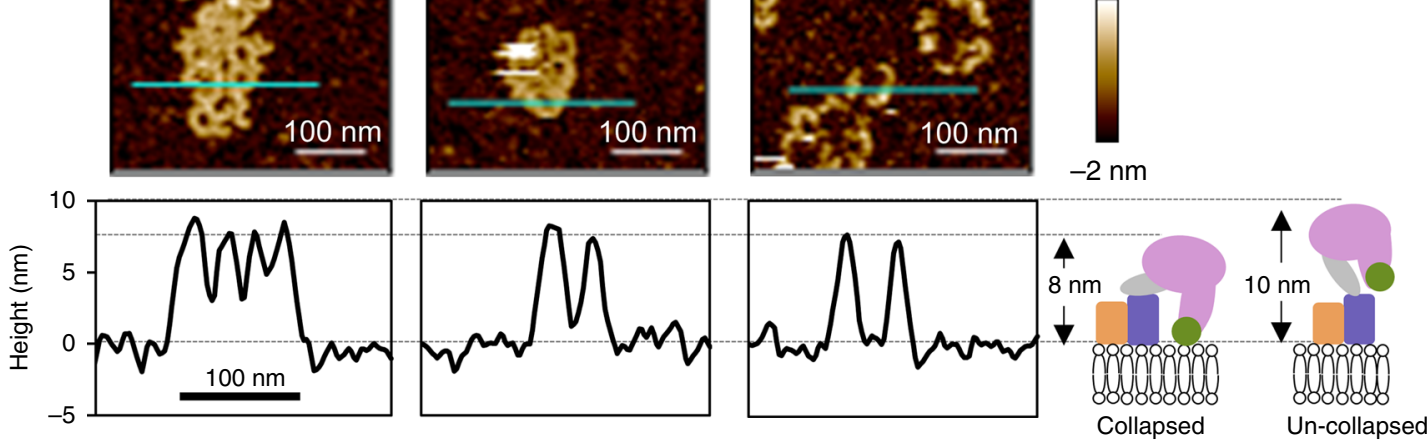

Fig. 2 Trapping pore formation of collapsed ILY oligomers. a Early prepore showing CD59 (orange) and ILY colored by domains: MACPF/CDC (pink), D2 (gray), D4 (purple). Domains 1 and 3 of ILY are grouped within the MACPF/CDC. HB1 (blue) and HB2 (green) are two helical bundles that form transmembrane $\beta$-hairpins in the final pore. Horizontal helix (h-helix) of HB2 is indicated (green arrow). Introduced cysteines (spheres) that form disulfidebonds to trap conformations: early prepore (red), HB1-lock (blue) and HB2-lock (green). b A fluorescence-based calcein-release assay was used to test lysis of liposomes containing cholesterol and CD59. ILY variants (wild-type (WT), early prepore, HB1-lock and HB2-lock) were analyzed for activity with and without reducing agent (DTT) pretreatment after $45 \mathrm{~min}$. The statistical significance of this comparison is indicated above a horizontal line. Statistical significance displayed above each -DTT bar is for comparisons against wild-type ILY without reducing agent. Individual data points from three independent experimental assays are shown as circles. Error bars represent standard deviation with the center of the bar indicating the mean value. $P$-value significance determined by 2-way ANOVA with a Bonferroni post-test: ns, not significant; ${ }^{\star} p<0.05$; ${ }^{\star \star} p<0.01$; ${ }^{* \star \star} p<0.001$. c AFM images (top) visualizing ILY variants on supported lipid bilayers containing cholesterol and CD59. Cyan line indicates image positions of height profiles (bottom) for ILY variants. White scale bar, $100 \mathrm{~nm}$. Average assembly height and standard deviation was measured from 10 such profiles for each ILY variant: HB1-lock (7.9

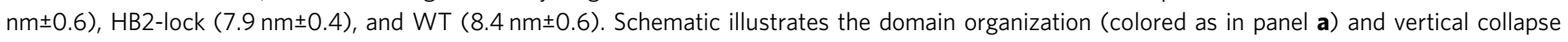
measurements for ILY conformations.

HB2 mutants remain unlocked. Despite the variable efficiency of disulfide bond formation both HB1-lock and HB2-lock mutants show a significant reduction in lytic activity, which is rescued under reducing conditions (Fig. 2b). Together these data suggest that variants could have a dominant negative effect on the lytic activity of an oligomer containing a mixture of locked and unlocked monomers. To investigate if these ILY variants formed vertically collapsed oligomers, we imaged complexes using atomic force microscopy on supported lipid bilayers containing both cholesterol and CD59. In contrast to monomeric ILY10 and the ILY early prepore (Fig. 2a), which extend $10 \mathrm{~nm}$ from the membrane surface, both HB1-lock and HB2-lock mutants are approximately $8 \mathrm{~nm}$ from the surface (Fig. $2 \mathrm{c}$ ). The vertical heights of the HB1-lock and HB2-lock oligomers are analogous to the $8 \mathrm{~nm}$ high wild-type pores (Fig. 2c), consistent with collapsed oligomers. Despite undergoing a vertical collapse, both mutants show significantly reduced lytic activity (Fig. 2b). We therefore conclude that HB1-lock and HB2-lock mutants trap collapsed prepore assemblies whereby the helical bundles are brought closer to the target membrane but do not breach the bilayer.
The h-helix is amphipathic, with charged or polar residues along the membrane-adjacent face and hydrophobic residues on the opposite face (Fig. 3a). To test how lytic activity and hence ILY function depend on the distribution of charges along the ILY h-helix, we generated ILY variants where charged or polar residues on the membrane-proximal face were substituted with alanine (ILYno charge) or where hydrophobic residues on the top face were swapped with lysine (ILYcharge+) (Fig. 3a). Removal of charged residues impaired lytic activity of wild-type ILY. By contrast, incorporating positively charged amino acids on the top face of the h-helix increases lysis. Lytic activity of wild-type ILY could also be improved by substituting h-helix residue N342 for tryptophan (Fig. 3b). Taken together, these results demonstrate that the lytic function of ILY can be modulated by changing the physicochemical properties of the h-helix.

Given that similar modifications did not impact lytic activity of the uncollapsed early prepore (Supplementary Fig. 6), we next analyzed the effect of h-helix variations on otherwise dysfunctional (non-lytic) collapsed prepores by introducing mutations in the HB1-lock and HB2-lock backgrounds. Remarkably, we found 
a

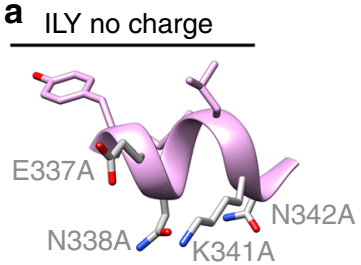

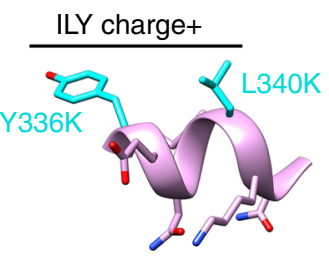

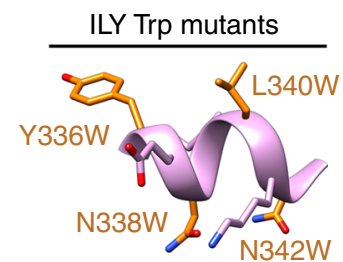

b

b DOPC:cholesterol liposomes

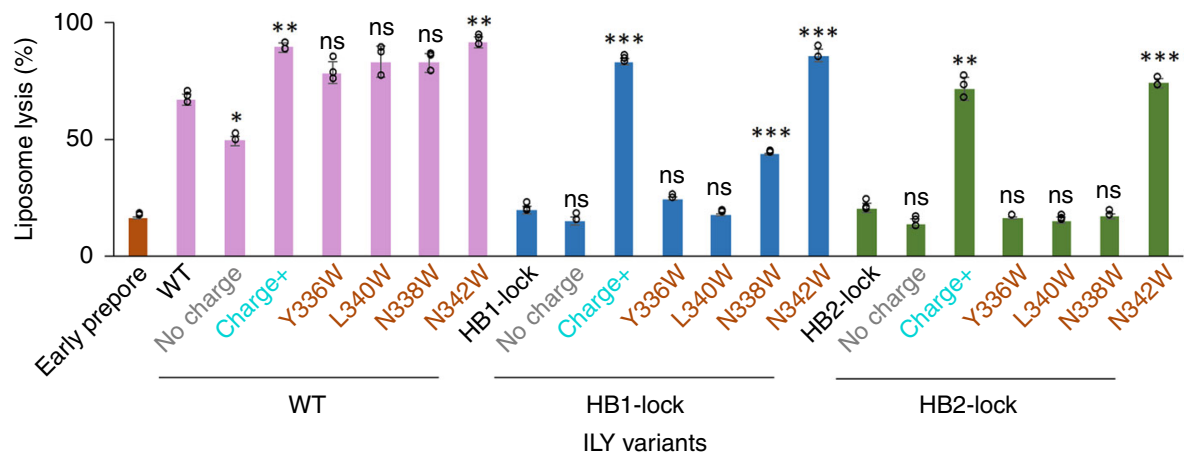

C DOPC liposomes

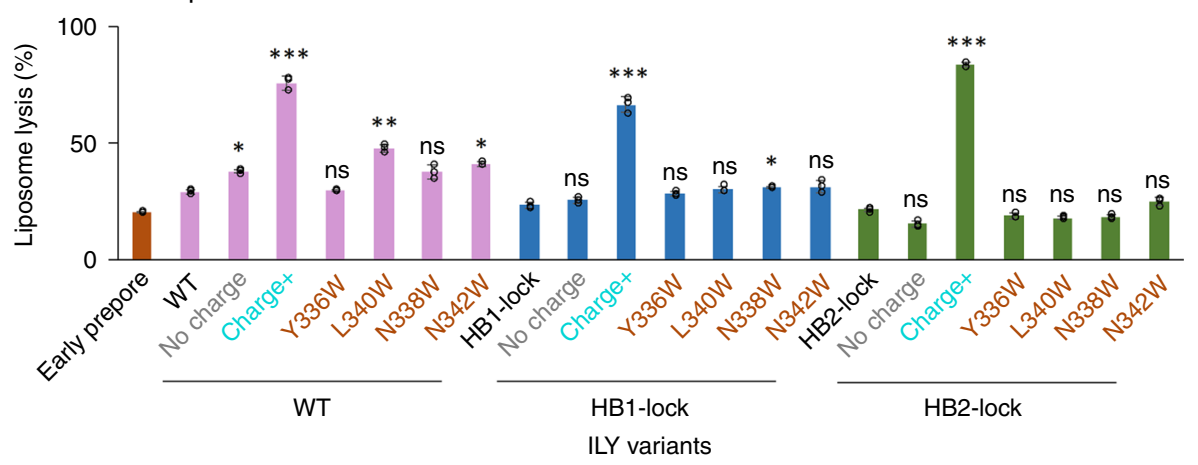

Fig. 3 Targeted mutations in the horizontal helix (h-helix) tune lytic activity of ILY. a H-helix mutations are mapped onto the ILY structure. Left panel (ILY no charge) highlights residues (gray) exchanged for alanine (E337A, N338A, K341A, N342A) on the membrane proximal face of the h-helix. Middle panel (ILY charge+) indicates residues (cyan) mutated to lysine (Y336K, L340K) on the top face of the helix. Right panel (ILY Trp mutants) shows the location of residues (orange) mutated to tryptophan (Y336W, N338W, L340W, or N342W). Sidechains have been added in COOT44 for visualization purposes. b, c Activity of ILY h-helix mutants in wild type (WT), HB1-lock or HB2-lock backgrounds were tested after 45 min using a calcein-release liposome lysis assay. Lysis of CD59-decorated liposomes comprised of DOPC:cholesterol lipids is shown in (b). Lysis of CD59-decorated DOPC liposomes is in (c). Statistical significance displayed above each bar is for comparison with the base ILY disulfide-locked variant (WT, HB1-lock, or HB2-lock, respectively). Individual data points from three independent experimental assays are shown as circles. Error bars represent standard deviation with the center of the bar indicating the mean value. P-value significance determined by one-way ANOVA with a Bonferroni post-test: $\mathrm{ns}$, not significant; ${ }^{*} p<0.05$; ${ }^{\star \star} p<0.01 ;{ }^{* \star \star} p<0.01$.

that without altering the proportion of disulfide-locked monomers (Supplementary Fig. 6), the lytic function was fully recovered by the addition of charged residues (charge + ) or the $\mathrm{N} 342 \mathrm{~W}$ substitution in the h-helix, as shown on cholesterolcontaining liposomes (Fig. 3b). By contrast, tryptophan substitutions elsewhere along the helix only partially recovered lytic activity for the HB1-lock mutant and did not enhance lysis at all for the HB2-lock mutant (Fig. 3b). We deduce that tryptophan substitutions along the bottom face of the h-helix augment lysis by intercalating into the outer leaflet of the bilayer and anchoring the h-helix to the membrane. The position of the tryptophan likely affects the efficiency of this mechanism, since substitution at N342 shows a stronger phenotypic change than at N338.

Since ILY only requires cholesterol to rupture the membrane and not to bind it ${ }^{10}$, the ILY-CD59 system offers an opportunity to explicitly investigate and potentially modulate the cholesteroldependency of CDC membrane lysis. We therefore measured the lytic activity of our ILY variants on liposomes without cholesterol (Fig. 3c). Notably, the requirement of cholesterol was abrogated by the addition of positively charged residues on the top face of the h-helix (charge+); this modification resulted in high levels of lysis in all ILY backgrounds (wild-type, HB1-lock, and HB2-lock). When the charges are removed from the h-helix of wild-type ILY, we found modest, but opposite changes in lysis of the two types of liposomes tested here (Fig. 3b, c), and no significant effect was observed in the HB1-lock or HB2-lock backgrounds. Furthermore, compared with the results on cholesterol-containing liposomes, N342W substitution in HB1-lock and HB2-lock backgrounds did not yield a similarly dramatic increase in lytic activity. Together, these data demonstrate that modifications of the h-helix can tune lytic activity in different lipid environments.

The amphipathic nature of the ILY h-helix is highly conserved across CDCs. Structures of both CD59-dependent (vaginolysin: VLY) and CD59-independent CDCs (pneumolysin: PLY, suilysin: 
a

Cholesterol-dependent cytolysins (CDCs)

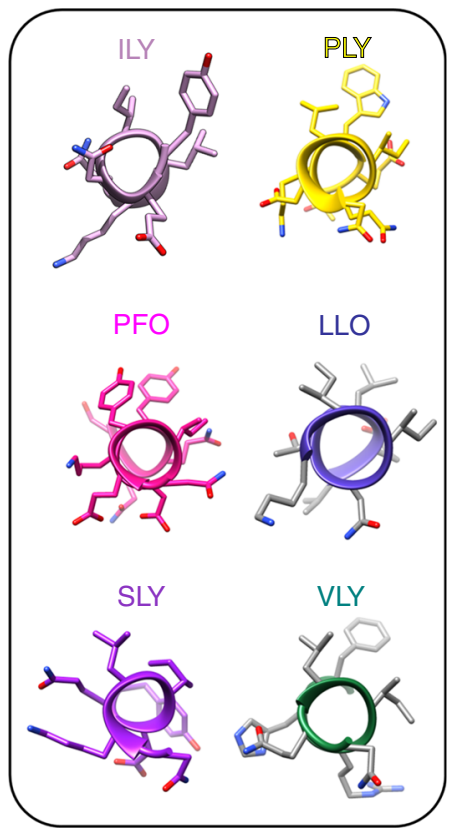

Other membranelysing toxins

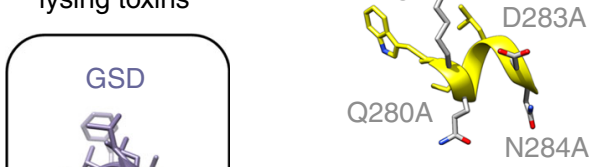

PLY charge+

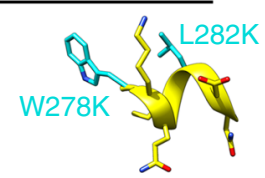

c

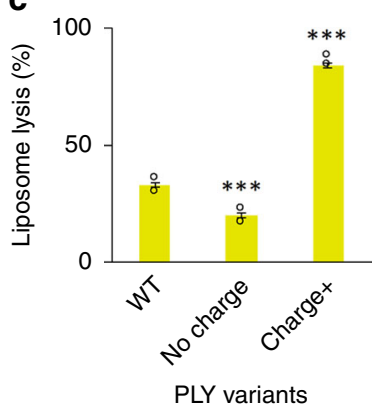

Fig. 4 H-helix modifications tune activity of a non-CD59-binding CDC. a The equivalent h-helix in cholesterol-dependent cytolysins (CDCs) are shown for intermedilysin (ILY, PDBID: 4BIK) 17 , pneumolysin (PLY, PDBID: 5AOE) ${ }^{15}$, perfringolysin O (PFO, PDBID: 1M3I) 49 , listeriolysin O (LLO, PDBID: 4CDB) 50 , suilysin (SLY, PDBID: $3 H V N)^{51}$, and vaginolysin (VLY, PDBID: $\left.5 I M Y\right)^{20}$. Amphipathic membrane-interacting helices are shown for other membrane-lysing proteins: gasdermin (GSD, PDBID: 6CB8) ${ }^{27}$, the CAMP cathelicidin (LL-37, PDBID: 5NMN) ${ }^{52}$, human beta-defensin 1 (HBD1, PDBID: $\left.11 \mathrm{JV}\right)^{53}$. b H-helix mutations in PLY analogous to the ILY variants are mapped onto the PLY structure (PDBID: 5AOE). The positions of alanine substitutions (K279A, Q280A, D283A, N284A) on the PLY h-helix are shown in gray (no charge). Residues on the PLY h-helix mutated to lysine (W278K, L282K) are highlighted in cyan (charge+). c The ability of PLY h-helix variants to lyse cholesterol-containing liposomes was assessed using a calcein-based lysis assay. Statistical significance displayed above each bar is for comparisons with wild-type PLY (WT). Individual data points from three independent experimental assays are shown as circles. Error bars represent standard deviation with the center of the bar indicating the mean value. P-value significance determined by one-way ANOVA with a Bonferroni post-test: ${ }^{\star \star \star} p<0.001$.

SLY, perfringolysin: PFO, and listeriolysin O: LLO) contain a similarly oriented amphipathic helix within HB2 (Fig. 4a). To test if the principles of tuning lytic activity can be extended to other CDCs, we introduced similar charge-altering mutations in PLY. As PLY requires cholesterol to initiate membrane attachment, we only used cholesterol-containing liposomes to compare lytic activity of PLY mutants with respect to wild-type PLY. In agreement with our results for ILY, PLY lytic activity was decreased when charged and polar amino acids of the h-helix were substituted with alanine (PLYno charge). Moreover, the lytic activity was enhanced by replacing uncharged residues on the top helical face with lysines (PLYcharge+ $)$, again analogous to what we observed for ILY variants (Fig. $4 \mathrm{~b}, \mathrm{c}$ ). These data suggest that modifications within the h-helix may provide a generic mechanism for controlling CDC pore formation and lytic activity.

\section{Discussion}

Our combined structural and biochemical data support a model whereby CDC oligomerization triggers movement of an amphipathic helix that modulates lytic activity (Fig. 5). Soluble CDC monomers associate with membranes through interactions within D4. Binding is mediated either by loops at the tip of D4 with cholesterol $^{7}$, or by engaging cell surface receptors, such as CD $59^{9}$. Membrane binding initiates oligomerization and conformational changes within the MACPF/CDC domain that create new interaction interfaces between monomers. Specifically, the formation of a helix-turn-helix motif exposes the MACPF/CDC $\beta-4$ strand to propagate the oligomer; it may also contribute to stability of the assembly. In our structure of an early prepore, the h-helices of ILY are arranged parallel to the membrane with their charged surfaces facing the outer leaflet of the lipid bilayer. Following a dramatic vertical collapse of the oligomeric prepore, the h-helix is brought in close proximity to the target membrane. Lying along the surface, the amphipathic h-helices of the oligomer would then cause local membrane disruption, analogous to the 'carpet' model of membrane disruption by cationic antimicrobial peptide $(\mathrm{CAMP})^{23}$ amphipathic helices (Fig. 4a). The helix-to-hairpin transition of CDC transmembrane residues is likely to occur via several intermediate structural states ${ }^{24}$ with different local energy minima, including collapsed coil-like intermediates ${ }^{25}$. The transient states of the h-helix may contribute to disruptive forces that prime the membrane for hairpin insertion and to the displacement of lipids by water molecules at the inner hydrophilic surface of the pore, as observed by molecular dynamics simulations ${ }^{26}$. We demonstrate that modifications of the h-helix similarly modulate the lytic activity of a non-CD59-binding CDC, PLY. Furthermore, amphipathic helices are present in and influence the lytic activity of other oligomeric pore-forming proteins such as gasdermin ${ }^{27}$ and MPEG-128,29 (Fig. 4a). Taken together, our model may reveal a fundamental mechanism underpinning membrane rupture by oligomeric pore-forming proteins.

Our results open an exciting perspective for protein engineering of h-helices for the creation of "super-CDCs" with 


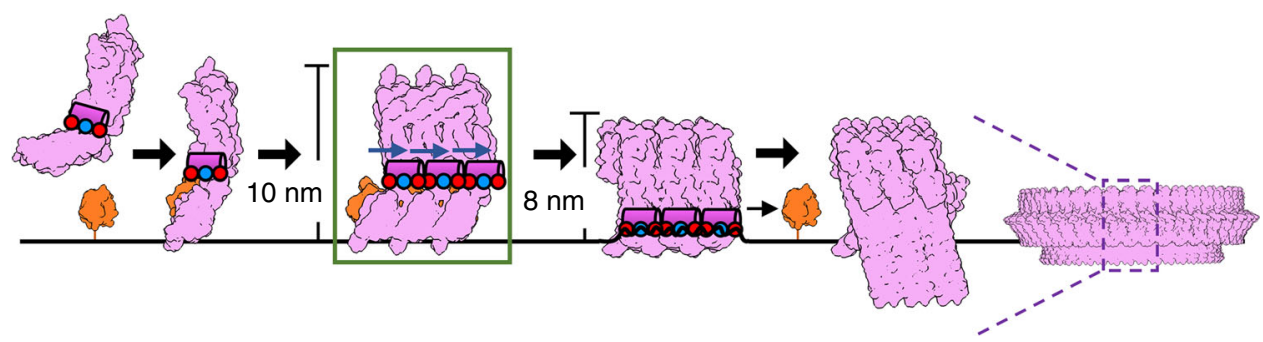

Fig. 5 Model for CDC pore formation and membrane lysis. Soluble monomers (pink) bind target membranes through interactions with either cholesterol or cell surface receptors, such as CD59 (orange). Oligomerization of the membrane-bound subunits (green box) causes structural rearrangements that include a shift (blue arrow) of the amphipathic h-helix (pink cylinder), as captured in our cryoEM reconstruction. The early prepore, which extends 10 nm from the lipid bilayer, collapses to $8 \mathrm{~nm}$ and brings the charged face of the h-helix (blue and red circles) into contact with the membrane. The amphipathic h-helix disrupts the membrane, leading to the transition of helical bundles (HB1 and HB2) into membrane-piercing $\beta$-hairpins. For ILY, CD59 dissociates, as it is not part of the final pore. Though only three monomers are displayed, this process occurs for higher oligomer arc and ring-like pores ${ }^{14}$. Figure is based on ILY and PLY structures: PDBIDs 6ZD0, 1S3R 54 , 4BIK17, 5CR6 15 , and 5LY6 ${ }^{15}$; and EMD-4118 15 .

significantly enhanced lytic activity or of cholesterol-independent CDCs that can be used to target bacterial pathogens. Specifically, our results show that CDC-mediated lysis is enhanced by the addition of positive charges to the top face of the h-helix, and that this removes the requirement of cholesterol for membrane rupture. We note that the tunable lysis of the h-helix bears similarities to CAMP-mediated cell lysis, where the membrane specificity is encoded by the charge properties of the helix ${ }^{30}$; and where the introduction of tryptophan residues can modulate antimicrobial activity of synthetic CAMPs ${ }^{31}$. By combining hhelix modifications with mutations in membrane-binding regions of $\mathrm{D} 4^{8,32,33}$, CDCs could be designed to lyse new cellular targets such as plant-pathogenic bacteria. The devastating effects of these pathogens on crop yield and productivity ${ }^{34}$ could be eliminated by engineered pore-forming proteins that lyse bacterial membranes but are unable to penetrate a plant cell wall.

\section{Methods}

Bacterial strains and plasmids. Escherichia coli strain DH5a was used to maintain plasmids and for cloning purposes. Strains BL21 DE3 and BL21 DE3 pLysS were used for expression of ILY and PLY variants, with the latter strain grown in the presence of $25 \mu \mathrm{g} / \mathrm{ml}$ chloramphenicol to maintain the pLysS plasmid. His-tagged ILY and PLY constructs were cloned and expressed using pTricHisA and maintained with $100 \mu \mathrm{g} / \mathrm{ml}$ ampicillin. His-tagged MSP2N2 ${ }^{35}$ was expressed from pET28-MSP2N2 (Addgene) in the presence of $50 \mu \mathrm{g} / \mathrm{ml}$ kanamycin.

Generation of ILY and PLY variant constructs. Wild-type ILY and PLY, for this study, have been codon optimized for $E$. coli and all native Cys in ILY have been mutated to $\operatorname{Ser}^{10}$. All ILY and PLY variants were created by site-directed mutagenesis, in which a pTrcHisA plasmid with the template gene was amplified with pairs of primers that contain the desired mutations (Supplementary Table 1) and Q5 DNA polymerase (New England Biolabs). This was followed by DpnI (New England Biolabs) digestion, to remove the template plasmid, heat-shock transformation into E. coli $\mathrm{DH} 5 \alpha$, and verification by sequencing. CDC variants used in this study are summarized in Supplementary Table 2.

Purification of ILY and PLY variants. E. coli BL21 DE3 containing pTrcHisA with an ily or ply variant gene were grown to an $\mathrm{OD}_{600}$ of $\sim 0.6-0.9$ in Luria-Bertani (LB) broth at $37^{\circ} \mathrm{C}$, and induced with a final concentration of $50 \mu \mathrm{g} / \mathrm{ml}$ of isopropyl $\beta$-d1-thiogalactopyranoside (IPTG) at $18^{\circ} \mathrm{C}$ for $18 \mathrm{~h}$. In the case of the over-active variants ILY-WT $\mathrm{T}^{\text {charge }+}$ and ILY-HB2lock ${ }^{\text {charge }+}$, this method resulted in very low levels of protein expression likely due to toxicity. Therefore, these two ILY variants were expressed in E. coli BL21 pLysS and induced with $50 \mu \mathrm{g} / \mathrm{ml}$ IPTG at $18^{\circ} \mathrm{C}$ for $1 \mathrm{~h}$. Regardless of the expression protocol, all variants were subjected to the same purification methods. The cells were collected by centrifugation and lysed by sonication in Buffer A ( $200 \mathrm{mM} \mathrm{NaCl}, 20 \mathrm{mM}$ Tris- $\mathrm{HCl}, \mathrm{pH}$ 7.5) containing cOmplete Protease Inhibitors (Roche). The soluble fraction was incubated with HisPur Cobalt resin (Thermo Fisher Scientific) and washed with $10 \mathrm{mM}$ imidazole in Buffer A. Bound His-tagged proteins were eluted with $100 \mathrm{mM}$ and $500 \mathrm{mM}$ imidazole in Buffer A. His-tagged protein was further purified by size exclusion chromatography over a Superdex $20010 / 300$ column (GE Healthcare) in Buffer A. The purity of all ILY and PLY variants was assessed by SDS-PAGE and their functionality was determined by the ability to form oligomers on lipid monolayers (Supplementary Fig. 6).
Forming the ILY early prepore on CD59-decorated lipid nanodiscs. MSP2N2 protein was first produced by growing BL21 DE3 cells containing pET28-MSP2N2 (Addgene) to an $\mathrm{OD}_{600}$ of $\sim 0.8$ in $\mathrm{LB}$ and inducing with $0.5 \mathrm{mM}$ IPTG at $37^{\circ} \mathrm{C}$ for $2-3 \mathrm{~h}$. Cells were pelleted and reconstituted in $40 \mathrm{mM}$ Tris- $\mathrm{HCl} \mathrm{pH} 7.4$ with cOmplete Protease Inhibitors (Roche), to which TritonX-100 was added to a final concentration of $1 \%$ and DNase I to a final concentration of $5 \mu \mathrm{g} / \mathrm{ml}$. Cells were stored on ice for $10 \mathrm{~min}$, then lysed by sonication and supplemented with $0.3 \mathrm{M}$ $\mathrm{NaCl}$. His-tagged protein was bound to HisPur Cobalt resin (Thermo Fisher Scientific), washed with $40 \mathrm{mM}$ Tris- $\mathrm{HCl} \mathrm{pH} 8.0,0.3 \mathrm{M} \mathrm{NaCl}, 0.5 \%$ Triton X-100, then washed with $40 \mathrm{mM}$ Tris- $\mathrm{HCl} \mathrm{pH} 8.0,0.3 \mathrm{M} \mathrm{NaCl}, 50 \mathrm{mM}$ sodium cholate. Protein was then eluted into $40 \mathrm{mM}$ Tris- $\mathrm{HCl} \mathrm{pH} 8.0,0.1 \mathrm{M} \mathrm{NaCl}, 300 \mathrm{mM}$ imidazole and subjected to size exclusion chromatography over a Superdex 200 10/300 column (GE Healthcare) with $40 \mathrm{mM}$ Tris- $\mathrm{HCl} \mathrm{pH} 8.0,0.1 \mathrm{M} \mathrm{NaCl}, 0.5 \mathrm{mM}$ EDTA. Nanodiscs were created by mixing $3.44 \mathrm{mM}$ MSP2N2 with $34.4 \mathrm{mM}$ lipids (DOPC:cholesterol, 1:1 molar ratio) solubilized in $40 \mathrm{mM}$ Tris- $\mathrm{HCl} \mathrm{pH} \mathrm{8.0,}$ $100 \mathrm{mM} \mathrm{NaCl}, 0.5 \mathrm{mM}$ EDTA, $64 \mathrm{mM}$ sodium cholate, such that the final sodium cholate concentration was $32.5 \mathrm{mM}$. This mixture was then incubated on ice for $30 \mathrm{~min}$ followed by an overnight incubation with activated Bio-beads SM2 (Bio$\mathrm{Rad}$ ) at $4{ }^{\circ} \mathrm{C}$ with agitation, to remove the detergent and promote nanodisc formation. The nanodiscs were then purified by size exclusion chromatography with $40 \mathrm{mM}$ Tris- $\mathrm{HCl} \mathrm{pH}$ 8.0, 0.1 M NaCl, $0.5 \mathrm{mM}$ EDTA over a Superose 6 10/300 column (GE Healthcare) and stored at $4{ }^{\circ} \mathrm{C}$.

A recombinant extracellular domain of CD59 modified with a lipid-anchoring peptide was gifted by R.A.G. Smith (King's College London). Briefly, the cytoplasmic domain of human CD59 modified with an additional C-terminal cysteine was expressed in E.coli and purified from inclusion bodies ${ }^{36}$. The cytotopic modification reagent bis-myristoyl lysyl SSKKSPSKKDDKKPGD (S-2thiopyridyl)-cysteine acid (APT3146, Cambridge Research Biochemicals) was covalently linked to the C-terminal cysteine and the modified protein was purified by hydrophobic interaction chromatography and ammonium sulfate precipitation $^{17,37}$.

Based on the peak absorbance reading for the nanodisc purification, $50 \mathrm{nM}$ MSP2N2 nanodiscs were incubated with $330 \mathrm{nM}$ CD59 at room temperature for $20 \mathrm{~min}$ in Buffer A, followed by another 20-min incubation after the addition of $20 \mu \mathrm{g} / \mathrm{ml}$ ILY-prepore. Assuming 100\% incorporation, each nanodisc likely contains 6 molecules of CD59; however, the size of the ILY oligomer may also be limited by the nanodisc diameter. The mixture was immediately washed twice with Buffer A on an Amicon Ultra $0.5 \mathrm{ml} 100 \mathrm{kDa}$ concentrator column (Merck) to remove unbound protein and concentrated by a factor of 5. To avoid particle aggregation, the final sample was immediately used to prepare EM grids.

Negative-stain EM. All EM samples were first screened by negative-stain EM, to assess particle integrity and distribution. $2.5 \mu \mathrm{l}$ of ILY-prepore-CD59 on nanodiscs was applied to glow-discharged, carbon-coated copper grids (Agar Scientific) and stained with $2 \%$ uranyl acetate. Samples were imaged on a $120 \mathrm{keV}$ Tecnai T12 microscope (Thermo Fisher Scientific) with a $2 \mathrm{~K}$ eagle camera (FEI) at a nominal magnification of $\times 50,000$ for evaluation.

CryoEM grid preparation and data collection. ILY-CD59 nanodisc complexes were imaged using holey carbon grids coated with graphene oxide. To coat R1.2/1.3 Quantifoil grids with graphene oxide, grids were first glow-discharged for $1 \mathrm{~min}$, then, $0.2 \mathrm{mg} / \mathrm{ml}$ of a graphene oxide solution (Sigma) in water was applied to the glow-discharged, top face of the grid, followed by blotting by filter paper on the bottom face of the grid. This process was repeated twice, followed by two washes of the top face of the grid with $20 \mu \mathrm{l}$ of water. Grids were left to dry, and used within one hour of graphene oxide coating. Immediately following concentration, $2.5 \mu$ of the early prepore-locked ILY-CD59 oligomers on nanodiscs was adsorbed on graphene oxide-coated grids and blotted for $2.5 \mathrm{~s}$ at "blot force" 3 and plunge 
frozen in liquid ethane cooled to liquid nitrogen temperatures with a Vitrobot mark IV (Thermo Fisher Scientific). Electron micrograph movies were collected on a $300 \mathrm{keV}$ Titan Krios (Thermo Fisher Scientific) fitted with a Falcon III direct electron detector (Thermo Fisher Scientific) in linear mode with image acquisition software EPU (Thermo Fisher scientific). Specific collection details for all three datasets are summarized in Supplementary Table 3.

Initial model generation. An initial model was generated in RELION ${ }^{19}$ from a dataset collected on a $300 \mathrm{keV}$ Titan Krios (Thermo Fisher Scientific) with a Quantum K2 Summit direct electron detector (Gatan) in counting mode with a magnified pixel size of $1.048 \AA$ A Manually picked particles from motion corrected and ctf-estimated micrographs were subjected to rounds of $2 \mathrm{D}$ classification and class curation, resulting in 4241 particles which were used to generate an initial model in RELION ${ }^{19}$. 3D refinement with this first initial model produced a reconstruction with an average resolution of $8.6 \AA$. This density was then used as the initial model for the first individual 3D reconstructions of datasets 1,2 , and 3 . Particles used to generate the initial model were not included in the refinement of subsequently collected datasets 1,2 , and 3 .

CryoEM data analysis and density reconstruction. The overall data analysis and reconstruction strategy are summarized in Supplementary Fig. 2. All data analysis and reconstruction were completed via RELION ${ }^{19}$ unless otherwise stated. Datasets 1,2 , and 3 were treated separately for the initial stages of data processing. For each dataset, micrograph movie frames were aligned using MotionCor ${ }^{38}$ and CTF parameters were estimated with CTFFIND4 ${ }^{39}$. Any flattened movies containing low figure of merit scores, crystalline ice, low contrast, or substantial drift were removed from further analysis. Particles were picked with a combination of manual picking, autopicking in RELION, and $\mathrm{crYOO}^{40}$, and duplicate particles were removed. Initial 2D class averages included signal from the nanodisc (Supplementary Fig. 1). Given the nature of the cytotopic linker, CD59 is likely flexibly tethered to the lipid nanodisc. To improve the alignment of the particles based on features of the ILY oligomer, particles were re-centered on the early prepore signal with a smaller mask to exclude the nanodisc. After several rounds of $2 \mathrm{D}$ classification and class selection, the remaining particles were reconstructed into $3 \mathrm{D}$ density using the initial model low pass filtered to $40 \AA$. The particles from dataset 3 were collected at a $30^{\circ}$ tilt to improve angular distribution of the particles and were therefore subjected to per-particle CTF refinement, followed by another round of auto-refinement. At this stage all particles from datasets 1, 2, and 3 were pooled, and after a final 2D classification, 105,448 particles were selected for 3D density reconstruction, resulting in an average resolution of $4.8 \AA$. To improve homogeneity in the particles, they were classified into 4 groups with local angular searches performed during each iteration. The combination of 2 classes $(51,041$ particles) produced the best quality density reconstruction, with an average resolution of $4.7 \AA$. Next, we implemented a local symmetry algorithm in RELION to average different regions of two adjacent monomers ${ }^{41}$. Adjacent monomers that exhibited the most contiguous density for helical regions in D1 after the $\mathrm{C} 1$ refinement were chosen for local symmetry averaging. We defined the top region (ILY MACPF/CDC domain and D2) and the bottom region (ILY D4 and CD59) with input masks (Supplementary Fig. 2). Implementing the local symmetry option for only the top region resulted in a reconstruction with the best local resolution and density features of the central monomer. The optimized transformation matrices are expressed as three Euler angles $(\theta$ 141.2, $\varphi 7.6$ and $\psi-134.0)$ and three translations $(\mathrm{X}-19.6 \AA, \mathrm{Y} 14.4 \AA$, Z $1.8 \AA$ ). The reconstruction was further refined with the same local symmetry designations, but in this case, references were low pass filtered by $10 \AA$ and only local searches were performed. The estimated resolution for the final density map is $4.6 \AA$ (Supplementary Fig. 1) with a global Bfactor of $-220 \AA^{2}$. Local resolution estimates were calculated using RELION and the local resolution-filtered map was used for building and refining the structural model. Maps were rendered using UCSF Chimera ${ }^{42}$.

Structural model building and refinement. To initiate refinement of the ILY early prepore transition state, we used a combination of templates comprised of the monomer-locked ILY-CD59 complex (PDBID 4BIK ${ }^{17}$ ) and the PLY pore model (PDBID 5LY6 ${ }^{43}$ ) (Supplementary Fig. 3). The crystal structure for soluble, monomer-locked ILY (PDBID 4BIK ${ }^{17}$ ) was placed in the middle monomer of the local resolution-filtered density with COOT ${ }^{44}$. The model was initially refined as 3 rigid bodies. The MACPF/CDC domain was segmented into domains: D1 and D3 as is convention. The first body comprised CD59 and ILY D4; the second was made up of D1 and D2; the third body was D3. The new helix-turn-helix motif in the $\mathrm{MACPF} / \mathrm{CDC}$ domain was built using the pneumolysin pore structure as a reference (PDBID 5LY ${ }^{15}$ ). The vertical and horizontal helices of HB2 were shifted into the density with the real space refine tool in $\mathrm{COOT}^{44}$. The helix-turn-helix motif and HB2 helices were further refined with global minimization and secondary structure restraint to generate the central monomer model in Phenix ${ }^{45}$. To generate the oligomeric structure of three ILY-CD59 subunits, the coordinates of the central monomer were rigid body fit into the neighboring densities using $\mathrm{COOT}^{44}$ and Phenix real_space_refine ${ }^{45}$. The oligomeric model was refined with Namdinator selecting the implicit solvent option, which combines Phenix real_space_refine with molecular dynamics simulations ${ }^{46}$. Sidechains were removed afterwards in
$\mathrm{COOT}^{44}$ and residue B-factors were refined using $\operatorname{Refmac}^{47}$, while maintaining coordinate positions. The final model is comprised of $88 \%$ of expressed residues and contains all amino acids present in the 4BIK template structure. The Phenix cryoEM validation tool ${ }^{48}$ was used to assess the validation statistics of the final model (Supplementary Table 4) and to generate average and per residue crosscorrelation coefficients comparing the map and model (Supplementary Fig. 4). The Map-Model FSC was calculated using Refmac and reported at a threshold of 0.5. For Figs. 1e and 3a, sidechains were added in COOT for visualization purposes only.

Fluorescence quenching assay. Fluorescently labeled ILY variants were generated by mutating L340 or N342 to a cysteine residue and covalent modification with monobromobimane $(\mathrm{mBBr})$. ILY variants were incubated with $\mathrm{mBBr}$ at $10^{\circ} \mathrm{C}$ overnight under agitation with a 1:5 molar ratio of ILY:mBBr in Buffer A. The free dye was removed by buffer exchange in Zeba spin $0.5 \mathrm{ml}$ columns (Thermo Fisher Scientific) and $\mathrm{mBBr}$-labeled protein, at a concentration of about $0.5 \mathrm{mg} / \mathrm{ml}$, was stored in the dark at $-80^{\circ} \mathrm{C}$ to preserve the fluorescent dye.

To test fluorescence of soluble, monomeric ILY, $9 \mu \mathrm{g} / \mathrm{ml}$ ILY-prepore- $\mathrm{mBBr}$ was mixed with $1.5 \mu \mathrm{g} / \mathrm{ml} \mathrm{CD59} \mathrm{in} \mathrm{Buffer} \mathrm{A;} \mathrm{and} \mathrm{for} \mathrm{the} \mathrm{oligomeric,} \mathrm{membrane-bound}$ condition, the ILY-prepore-mBBr and CD59 mixture also included $0.375 \mu \mathrm{g} / \mathrm{ml}$ of DOPC:cholesterol (1:1 molar ratio) liposomes ${ }^{6}$. Fluorescence was measured by excitation at $398 \mathrm{~nm}$, and an emission spectrum was collected from $430-600 \mathrm{~nm}$ on a CLARIOstar plate reader (BMG labtech), with the spectrum peak taken as the emission fluorescence reading. Fluorescence of each sample was normalized to the fluorescence of denatured protein (addition of $10 \%$ SDS) by dividing the monomer or prepore fluorescence by the denatured fluorescence value of each respective sample. Plots were generated using Microsoft Excel.

Gel shift assay. Disulfide bond formation was assessed by incubating $45 \mu \mathrm{g} / \mathrm{ml}$ of each ILY variant with reducing $(62.5 \mathrm{mM}$ Tris $\mathrm{pH} 6.5,10 \%$ glycerol, $0.05 \%$ bromophenol blue, $2 \%$ SDS, $75 \mathrm{mM}$ DTT, final concentrations) or non-reducing $(62.5$ $\mathrm{mM}$ Tris pH 6.5, 10\% glycerol, $0.05 \%$ bromophenol blue, $2 \%$ SDS, final concentrations) sample buffer at $90^{\circ} \mathrm{C}$ for $5 \mathrm{~min}$. $225 \mathrm{ng}$ of each ILY variant was analyzed by running $4-12 \%$ BisTris NuPAGE gels (Thermo Fisher Scientific) with Bolt MOPS SDS Running Buffer (Life Technologies). Gels were then stained with Quick coomassie stain (Generon) and destained in water overnight before imaging.

Lipid monolayer preparation and negative staining. Integrity and folding of all ILY variants were assessed by ring and arc formation on lipid monolayers. $10 \mu \mathrm{g} / \mathrm{m}$ ILY and $1.6 \mu \mathrm{g} / \mathrm{ml} \mathrm{CD59,} \mathrm{final} \mathrm{concentrations,} \mathrm{were} \mathrm{mixed} \mathrm{in} \mathrm{Buffer} \mathrm{A} \mathrm{in} \mathrm{a} \mathrm{multi-}$ well Teflon plate. The protein mixture was then overlaid with $2 \mu \mathrm{l}$ of chloroform containing $1 \mathrm{mg} / \mathrm{ml}$ DOPC:cholesterol (1:1 molar ratio) and incubated at room temperature for $2 \mathrm{~min}$ to allow chloroform evaporation. Carbon-coated copper grids (Agar Scientific) were applied to the top of each well before incubattion at $37^{\circ} \mathrm{C}$ for $15 \mathrm{~min}$ in a sealed petri dish with a Buffer A-soaked paper towel, to prevent evaporation. Grids were then stained with $2 \%$ uranyl acetate and imaged, as described earlier (Negative-stain EM).

Liposome lysis assay. Lytic activity of $\mathrm{CDC}$ variants was assessed using a calceinrelease liposome lysis assay. Liposomes containing calcein were first prepared by rehydrating lipids (DOPC or DOPC:cholesterol, 1:1 molar ratio) in Buffer A with $50 \mathrm{mM}$ calcein followed by extrusion through a $0.1 \mu \mathrm{m}$ filter. Unilamellar calceincontaining liposomes were purified by size exclusion chromatography with Sephadex G-50 resin (Sigma) in Buffer A with $500 \mathrm{mM}$ sucrose, as previously described ${ }^{10}$. The resultant liposomes were filled with self-quenching calcein dye while external, unquenched dye was replaced by Buffer A and sucrose. To determine the activity of ILY variants, liposomes were first incubated with a final concentration of $1.0 \mu \mathrm{g} / \mathrm{ml} \mathrm{CD} 59$ for $20 \mathrm{~min}$ at room temperature, followed by the addition of ILY at a final concentration of $9.0 \mu \mathrm{g} / \mathrm{ml}$. The activity of PLY variants was determined by adding a final concentration of $48.4 \mu \mathrm{g} / \mathrm{ml}$ of PLY to DOPC: cholesterol liposomes. Fluorescence intensity was read with a CLARIOstar plate reader (BMG labtech) at an excitation wavelength of $490 \mathrm{~nm}$ and emission wavelength of $520 \mathrm{~nm}$. Total liposomes lysis was achieved by the addition of $0.87 \%$ n-dodecyl-beta-maltoside (DDM) and a freeze/thaw cycle at $-80^{\circ} \mathrm{C}$. To calculate percent lysis, the fluorescence value from a buffer well was first subtracted from all raw fluorescence readings at a single time point between 30 and $60 \mathrm{~min}$. Each blanked-corrected experimental reading was divided by the corresponding total liposome lysis fluorescence reading (also blank-corrected). Activity of disulfidelocked CDC variants was assessed by preincubating the protein with or without $20 \mathrm{mM}$ DTT for $30 \mathrm{~min}$ at room temperature.

Atomic force microscopy sample preparation. For the preparation of supported lipid bilayers, pure lipids were dissolved in chloroform at $10 \mathrm{mg} / \mathrm{mL}$ and mixed in solution to give a lipid mixture of DOPC:cholesterol 2:1 molar ratio. The lipid-inchloroform solution was then dried in a glass vial under a stream of nitrogen gas to give $1 \mathrm{mg}$ of lipid as a thin film. The lipid film was hydrated in buffer $(20 \mathrm{mM}$ Tris, $200 \mathrm{mM} \mathrm{NaCl}, \mathrm{pH} 7.5$ ), vortexed and bath sonicated to give a cloudy lipid suspension. The suspension was then passed through a $50 \mathrm{~nm}$ polycarbonate 
membrane (GE Healthcare Lifesciences) 15 times to yield a clear suspension of small unilamellar vesicles.

Supported lipid bilayers were formed by injecting $4.5 \mu \mathrm{L}$ of the lipid vesicle suspension to a freshly cleaved mica disk $(6 \mathrm{~mm}$ diameter) under $18 \mu \mathrm{L}$ of incubation buffer (hydration buffer plus $10 \mathrm{mM} \mathrm{CaCl} 2$ solution) at $37^{\circ} \mathrm{C}$; this induces the rupture of the vesicles onto the mica support over an incubation period of approximately $30 \mathrm{~min}$. Excess vesicles were then removed from the supernatant by rinsing with $500 \mu \mathrm{L}$ of the hydration buffer, to yield a uniform bilayer free of adsorbed vesicles (as assessed by AFM imaging). The supported lipid bilayers were next incubated with a final concentration of $50 \mathrm{ng} / \mathrm{ml} \mathrm{CD} 59$ for $5 \mathrm{~min}$, and thereafter with a final concentration of $100 \mu \mathrm{g} / \mathrm{ml} \mathrm{ILY}$ for $15 \mathrm{~min}$, all at $37^{\circ} \mathrm{C}$, and next washed with $500 \mu \mathrm{L}$ of the hydration buffer.

Atomic force microscopy imaging and analysis. AFM imaging was performed in buffer solution using a Multimode 8 AFM (Bruker, Santa Barbara, USA) and MSNL-E and PEAKFORCE-HIRS-F-B cantilevers (Bruker), in off-resonance tapping/fast force-feedback imaging (Bruker's PeakForce Tapping) mode where forcedistance curves were recorded at $2 \mathrm{kHz}$, with amplitudes of $10-20 \mathrm{~nm}$, with the maximum imaging forces estimated between 50 and $100 \mathrm{pN}$. The experiments were performed at room temperature. Images were typically recorded at a few minutes per frame on an E scanner (Bruker, Santa Barbara, CA, USA).

We note that (membrane-inserted) pore assemblies are readily imaged by AFM, as they make contact, through the membrane, with the underlying solid support; but that prepore assemblies are rather hard to image by AFM. Loosely packed assemblies may be broken apart under the mechanical stress of the AFM tip. In addition, the supported lipid bilayers generally represent too fluid a support to retain the assemblies in place ${ }^{3}$, except when the assemblies form larger clusters. In this case, the mobility of HB1/HB2-lock prepore assemblies may also be constrained by the dense coverage of CD59 on the membrane surface ${ }^{10}$.

Images were processed using open-source SPM analysis software, Gwyddion $(\mathrm{v} 2.55)^{43}$ for first-order plane-fit background subtraction and second-order lineby-line flattening. The membrane surface was referenced as zero height. The images in Fig. 2c were cropped from $500 \times 500 \mathrm{~nm}^{2}$. Assembly heights (mean \pm standard deviation) were estimated based on 10 height profiles for 10 different assemblies from each $500 \times 500 \mathrm{~nm}^{2}$ image, referenced with respect to the membrane surface, as exemplified in Fig. 2c.

Statistical analysis. The Student's $t$ test (assuming equal variance) was used to assess statistical significance of differences between monomer and early prepore fluorescence quenching of h-helix-labeled ILY (ILY-prepore ${ }^{\mathrm{N} 342 \mathrm{C}}$ or ILYprepore ${ }^{\mathrm{L} 340 \mathrm{C}}$ ). To compare the lytic activity of disulfide-locked mutants in the presence or absence of DTT, first a two-factor ANOVA test was performed to confirm significant variance amongst the samples, followed by a Bonferroni posttest for paired comparisons. A single-factor ANOVA test was used to determine the significance of variance within datasets describing lytic activity of h-helix mutants (ILY and PLY). A Bonferroni post-test was used for paired comparisons within each set of h-helix variants (ILY-WT, ILY-HB1lock, ILY-HB2lock, PLY).

Reporting summary. Further information on research design is available in the Nature Research Reporting Summary linked to this article.

\section{Data availability}

Data supporting the findings of this manuscript are available from the corresponding authors upon reasonable request. All ILY and PLY mutants created in this study are made available at Addgene. A reporting summary for this article is available as a Supplementary Information file. The accession numbers for the EM map and model of the ILY-CD59 early prepore complex reported in this paper are EMD 11172 and PDB $6 Z D 0$. Source data are provided with this paper.

Received: 29 June 2020; Accepted: 14 October 2020;

Published online: 16 November 2020

\section{References}

1. Gonzalez, M. R., Bischofberger, M., Pernot, L., van der Goot, F. G. \& Frêche, B. Bacterial pore-forming toxins: the (w)hole story? Cell. Mol. Life Sci. 65, 493-507 (2008).

2. Dal Peraro, M. \& van der Goot, F. G. Pore-forming toxins: ancient, but never really out of fashion. Nat. Rev. Microbiol. 14, 77-92 (2016).

3. Rudd-Schmidt, J. A. et al. Lipid order and charge protect killer T cells from accidental death. Nat. Commun. 10, 5396 (2019).

4. Ding, J. et al. Pore-forming activity and structural autoinhibition of the gasdermin family. Nature 535, 111-116 (2016).

5. Broz, P., Pelegrín, P. \& Shao, F. The gasdermins, a protein family executing cell death and inflammation. Nat. Rev. Immunol. 20, 143-157 (2020).
6. Tweten, R. K. Cholesterol-dependent cytolysins, a family of versatile poreforming toxins. Infect. Immun. 73, 6199-6209 (2005).

7. Farrand, A. J., LaChapelle, S., Hotze, E. M., Johnson, A. E. \& Tweten, R. K. Only two amino acids are essential for cytolytic toxin recognition of cholesterol at the membrane surface. Proc. Natl Acad. Sci. USA 107, 4341-4346 (2010).

8. Subramanian, K. et al. Pneumolysin binds to the mannose receptor C type 1 (MRC-1) leading to anti-inflammatory responses and enhanced pneumococcal survival. Nat. Microbiol. 4, 62-70 (2019).

9. Giddings, K. S., Zhao, J., Sims, P. J. \& Tweten, R. K. Human CD59 is a receptor for the cholesterol-dependent cytolysin intermedilysin. Nat. Struct. Mol. Biol. 11, 1173-1178 (2004).

10. Boyd, C. M. et al. Disentangling the roles of cholesterol and CD59 in intermedilysin pore formation. Sci. Rep. 6, 38446 (2016).

11. Lukoyanova, N., Hoogenboom, B. W. \& Saibil, H. R. The membrane attack complex, perforin and cholesterol-dependent cytolysin superfamily of poreforming proteins. J. Cell Sci. 129, 2125-2133 (2016).

12. Shepard, L. A. et al. Identification of a membrane-spanning domain of the thiol-activated pore-forming toxin Clostridium perfringens perfringolysin $\mathrm{O}$ : an alpha-helical to beta-sheet transition identified by fluorescence spectroscopy. Biochemistry 37, 14563-14574 (1998).

13. Shepard, L. A., Shatursky, O., Johnson, A. E. \& Tweten, R. K. The mechanism of pore assembly for a cholesterol-dependent cytolysin: formation of a large prepore complex precedes the insertion of the transmembrane beta-hairpins. Biochemistry 39, 10284-10293 (2000).

14. Leung, C. et al. Stepwise visualization of membrane pore formation by suilysin, a bacterial cholesterol-dependent cytolysin. eLife 3, e04247 (2014).

15. van Pee, K. et al. CryoEM structures of membrane pore and prepore complex reveal cytolytic mechanism of Pneumolysin. eLife 6, e23644 (2017).

16. Wade, K. R. et al. An intermolecular electrostatic interaction controls the prepore-to-pore transition in a cholesterol-dependent cytolysin. Proc. Natl Acad. Sci. USA 112, 2204-2209 (2015).

17. Johnson, S., Brooks, N. J., Smith, R. A. G., Lea, S. M. \& Bubeck, D. Structural basis for recognition of the pore-forming toxin intermedilysin by human complement receptor CD59. Cell Rep. 3, 1369-1377 (2013).

18. LaChapelle, S., Tweten, R. K. \& Hotze, E. M. Intermedilysin-receptor interactions during assembly of the pore complex: assembly intermediates increase host cell susceptibility to complement-mediated lysis. J. Biol. Chem. 284, 12719-12726 (2009).

19. Zivanov, J. et al. New tools for automated high-resolution cryo-EM structure determination in RELION-3. eLife 7, e42166 (2018).

20. Lawrence, S. L. et al. Structural basis for receptor recognition by the human CD59-responsive cholesterol-dependent cytolysins. Structure 24, 1488-1498 (2016).

21. Rudd, P. M. et al. The glycosylation of the complement regulatory protein, human erythrocyte CD59. J. Biol. Chem. 272, 7229-7244 (1997).

22. Shewell, L. K. et al. All major cholesterol-dependent cytolysins use glycans as cellular receptors. Sci. Adv. 6, eaaz4926 (2020).

23. Pouny, Y., Rapaport, D., Mor, A., Nicolas, P. \& Shai, Y. Interaction of antimicrobial dermaseptin and its fluorescently labeled analogs with phospholipid membranes. Biochemistry 31, 12416-12423 (1992).

24. Lukoyanova, N. et al. Conformational changes during pore formation by the perforin-related protein pleurotolysin. PLoS Biol. 13, e1002049 (2015).

25. Chakraborty, D., Chebaro, Y. \& Wales, D. J. A multifunnel energy landscape encodes the competing $\alpha$-helix and $\beta$-hairpin conformations for a designed peptide. Phys. Chem. Chem. Phys. 22, 1359-1370 (2020).

26. Vögele, M. et al. Membrane perforation by the pore-forming toxin pneumolysin. Proc. Natl Acad. Sci. USA 116, 13352-13357 (2019).

27. Ruan, J., Xia, S., Liu, X., Lieberman, J. \& Wu, H. Cryo-EM structure of the gasdermin A3 membrane pore. Nature 557, 62-67 (2018).

28. Pang, S. S. et al. The cryo-EM structure of the acid activatable pore-forming immune effector Macrophage-expressed gene 1. Nat. Commun. 10, 4288 (2019).

29. $\mathrm{Ni}, \mathrm{T}$. et al. Structure and mechanism of bactericidal mammalian perforin-2 an ancient agent of innate immunity. Sci. Adv. 6, eaax8286 (2020).

30. Dathe, M., Nikolenko, H., Meyer, J., Beyermann, M. \& Bienert, M. Optimization of the antimicrobial activity of magainin peptides by modification of charge. FEBS Lett. 501, 146-150 (2001).

31. Bi, X., Wang, C., Ma, L., Sun, Y. \& Shang, D. Investigation of the role of tryptophan residues in cationic antimicrobial peptides to determine the mechanism of antimicrobial action. J. Appl. Microbiol. 115, 663-672 (2013).

32. Farrand, A. J. et al. The cholesterol-dependent cytolysin membrane-binding interface discriminates lipid environments of cholesterol to support $\beta$-barrel pore insertion. J. Biol. Chem. 290, 17733-17744 (2015).

33. Shewell, L. K. et al. The cholesterol-dependent cytolysins pneumolysin and streptolysin $\mathrm{O}$ require binding to red blood cell glycans for hemolytic activity. Proc. Natl Acad. Sci. USA 111, E5312-E5320 (2014).

34. Abramovitch, R. B., Anderson, J. C. \& Martin, G. B. Bacterial elicitation and evasion of plant innate immunity. Nat. Rev. Mol. Cell Biol. 7, 601-611 (2006). 
35. Grinkova, Y. V., Denisov, I. G. \& Sligar, S. G. Engineering extended membrane scaffold proteins for self-assembly of soluble nanoscale lipid bilayers. Protein Eng. Des. Sel. 23, 843-848 (2010).

36. Leath, K. J. et al. High-resolution structures of bacterially expressed soluble human CD59. Acta Crystallogr. F. 63, 648-652 (2007).

37. Fraser, D. A. et al. Generation of a recombinant, membrane-targeted form of the complement regulator CD59: activity in vitro and in vivo. J. Biol. Chem. 278, 48921-48927 (2003).

38. Zheng, S. Q. et al. MotionCor2: anisotropic correction of beam-induced motion for improved cryo-electron microscopy. Nat. Methods 14, 331-332 (2017).

39. Rohou, A. \& Grigorieff, N. CTFFIND4: Fast and accurate defocus estimation from electron micrographs. J. Struct. Biol. 192, 216-221 (2015).

40. Wagner, T. et al. SPHIRE-crYOLO is a fast and accurate fully automated particle picker for cryo-EM. Commun. Biol. 2, 218 (2019).

41. Shakeel, S. et al. Structure of the Fanconi anaemia monoubiquitin ligase complex. Nature 575, 234-237 (2019).

42. Pettersen, E. F. et al. UCSF Chimera-a visualization system for exploratory research and analysis. J. Comput. Chem. 25, 1605-1612 (2004).

43. Nečas, D. \& Klapetek, P. Gwyddion: an open-source software for SPM data analysis. Cent. Eur. J. Phys. 10, 181-188 (2012).

44. Emsley, P., Lohkamp, B., Scott, W. G. \& Cowtan, K. Features and development of Coot. Acta Crystallogr. D 66, 486-501 (2010).

45. Afonine, P. V. et al. Real-space refinement in PHENIX for cryo-EM and crystallography. Acta Crystallogr. D 74, 531-544 (2018).

46. Kidmose, R. T. et al. Namdinator-automatic molecular dynamics flexible fitting of structural models into cryo-EM and crystallography experimental maps. IUCrJ 6, 526-531 (2019).

47. Murshudov, G. N. et al. REFMAC5 for the refinement of macromolecular crystal structures. Acta Crystallogr. D 67, 355-367 (2011).

48. Afonine, P. V. et al. New tools for the analysis and validation of cryo-EM maps and atomic models. Acta Crystallogr. D 74, 814-840 (2018).

49. Rossjohn, J. et al. Structures of perfringolysin O suggest a pathway for activation of cholesterol-dependent cytolysins. J. Mol. Biol. 367, 1227-1236 (2007).

50. Köster, S. et al. Crystal structure of listeriolysin O reveals molecular details of oligomerization and pore formation. Nat. Commun. 5, 3690 (2014).

51. Xu, L. et al. Crystal structure of cytotoxin protein suilysin from Streptococcus suis. Protein Cell 1, 96-105 (2010).

52. Sancho-Vaello, E. et al. Structural remodeling and oligomerization of human cathelicidin on membranes suggest fibril-like structures as active species. Sci. Rep. 7, 15371 (2017).

53. Hoover, D. M., Chertov, O. \& Lubkowski, J. The structure of human betadefensin-1: new insights into structural properties of beta-defensins. J. Biol. Chem. 276, 39021-39026 (2001).

54. Polekhina, G., Giddings, K. S., Tweten, R. K. \& Parker, M. W. Insights into the action of the superfamily of cholesterol-dependent cytolysins from studies of intermedilysin. Proc. Natl Acad. Sci. USA 102, 600-605 (2005).

\section{Acknowledgements}

The authors thank R. Smith for gifting the CD59; Y. Chaban for data acquisition assistance; S. Islam for computational support; P. Simpson for EM support; J. Demmer for assistance with model building; A.J. Praveen for model validation advice; P. Haynes for guidance on the AFM image analysis; and A. Menny and the Bubeck lab for discussions. We thank Diamond for access and support of the Cryo-EM facilities at the UK national electron bio-imaging center (eBIC), proposal EM18659, funded by the Well come Trust, MRC, and BBSRC. D.B. and N.R.S. are supported by a CRUK Career Establishment Award (C26409/A16099) to D.B.; C.M.B. and T.B.V. are funded by BBSRC Doctoral Training Program grants, Ref: BB/J014575/1 and BB/M011178/1, respectively. E.S.P. and B.W.H. have been supported by EPSRC and MRC (EP/M507970/ 1 to E.S.P.; MR/R000328/1 to B.W.H.), and acknowledge EPSRC investment in AFM equipment (EP/M028100/1).

\section{Author contributions}

N.R.S. conducted cryoEM work, generated ILY mutants, and performed ILY fluorescence and lysis experiments. N.R.S. and D.B. conceived the ideas, analyzed the results, and wrote the manuscript. T.B.V. generated PLY mutants and ILY-prepore ${ }^{\text {charge+ }}{ }^{\text {, conducted }}$ PLY and ILY-prepore ${ }^{\text {charge }+}$ lysis experiments, analyzed these data, and prepared figures. T.B.V., E.S.P., and B.W.H. analyzed AFM data. E.S.P. performed AFM experiments. C.M.B. generated the ILY-HB1lock mutant. All authors assisted with manuscript editing.

\section{Competing interests}

The authors declare no competing interests.

\section{Additional information}

Supplementary information is available for this paper at https://doi.org/10.1038/s41467020-19482-6.

Correspondence and requests for materials should be addressed to D.B.

Peer review information Nature Communications thanks Gregor Anderluh, Robert Gilbert, and Michael Landsberg for their contributions to the peer review of this work.

Reprints and permission information is available at http://www.nature.com/reprints

Publisher's note Springer Nature remains neutral with regard to jurisdictional claims in published maps and institutional affiliations.

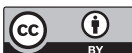

Open Access This article is licensed under a Creative Commons Attribution 4.0 International License, which permits use, sharing, adaptation, distribution and reproduction in any medium or format, as long as you give appropriate credit to the original author(s) and the source, provide a link to the Creative Commons license, and indicate if changes were made. The images or other third party material in this article are included in the article's Creative Commons license, unless indicated otherwise in a credit line to the material. If material is not included in the article's Creative Commons license and your intended use is not permitted by statutory regulation or exceeds the permitted use, you will need to obtain permission directly from the copyright holder. To view a copy of this license, visit http://creativecommons.org/ licenses/by/4.0/.

(C) The Author(s) 2020 